\title{
A QFT ROBUST CONTROLLER AS A REMEDY FOR TRMS
}

\author{
Mostafa HONARI-TORSHIZI (D) ${ }^{1 *}$, Hossein RAHMANI (i) ${ }^{2}$, Hossein MOEINKHAH (D) ${ }^{3}$, \\ Mohammad Reza GHARIB (1D) ${ }^{4 *}$, Javad JAHANPOUR (D) 5 \\ 1,2,3 Department of Mechanical Engineering, University of Sistan and Baluchestan, Zahedan, Iran \\ ${ }^{4}$ Department of Mechanical Engineering, University of Torbat Heydarieh, Torbat Heydarieh, Iran \\ ${ }^{5}$ Department of Mechanical Engineering, Mashhad Branch, Islamic Azad University, Mashhad, Iran
}

Received 22 November 2019; accepted 05 February 2020

\begin{abstract}
Control of a Twin Rotor Multi-input Multi-output System (TRMS) is not a simple work. Because it has complex nonlinear dynamics, cross-coupling, uncertainties, and instability. This paper provides a practical method for control of a TRMS, named Quantitative Feedback Theory (QFT) as one of the robust approaches. Firstly, the TRMS set and modeling procedure are introduced. Secondly, the nonlinear and linear equations of electrical and mechanical parts in both vertical and horizontal planes are presented. Next, using the QFT method, a controller is designed for motion in each plane. Finally, the robustness of the control strategy is illustrated by simulations of vertical and horizontal motions, including controller and pre-filter in the presence of uncertainties. The results demonstrate that the proposed robust controller can guarantee the system stabilization, as well as pitch and yaw tracking of TRMS.
\end{abstract}

Keywords: QFT, robust control, TRMS, uncertainty, simulation, cross-coupling.

\section{Introduction}

Over the years, many control techniques for designing the flight control system of manned and unmanned helicopters have been extensively studied (Oktay et al., 2016; Oktay \& Sal, 2015, 2016). Vilchis et al. (2003), stated that the main difficulties for designing stable feedback controllers for helicopters stem from their nonlinearities and couplings. In certain aspects, TRMS behavior resembles that of a helicopter and inherits a challenging engineering problem due to its high nonlinearity, the cross-coupling between its two axes, instability, complex dynamics and inaccessibility of some of its states and outputs for measurements (Choudhary, 2017; Feedback Instruments Ltd, 1998; Feedback Instruments Ltd, 2006; Gorczyca \&
Hajduk, 2004; Juang et al., 2008; Juang et al., 2011; Raghavan \& Thomas, 2017; Rahideh et al., 2007; Rahideh et al., 2008; Tao et al., 2010; Wen \& Lu, 2008). However, the TRMS is substantially different from a helicopter ( $\mathrm{Ta}$ ble 1). The TRMS is a laboratory platform developed by Feedback Instruments Ltd. (1998) for control experiments in universities and research centers. It has a beam (consist of a main and tail beam) pivoted on its base in such a way that it can rotate freely, resulting in pitch $\left(\theta_{v}\right)$ and azimuth angles $\left(\theta_{h}\right)$ measured by position sensors fitted at the pivot (Figure 1). At both ends of the beam, there are two rotors (the main and tail rotors) driven by DC-motors generating the vertical and horizontal thrusts. The aerodynamic forces are controlled by varying the speed of the motors. Two corresponding angular velocities of the rotors are

Table 1. The main differences between a helicopter and a TRMS (Rahideh et al., 2008)

\begin{tabular}{|l|c|c|}
\hline & TRMS & Helicopter \\
\hline Location of the pivot point & Midway between two rotors & The main rotor head \\
\hline Lift generation or vertical control & Speed control of the main rotor & Collective pitch control \\
\hline Yaw is controlled by & Tail rotor speed & Pitch angle of the tail rotor blades \\
\hline Cyclical control & No & Yes (for directional control) \\
\hline
\end{tabular}

${ }^{*}$ Corresponding author. E-mail: m.gharib@torbath.ac.ir 


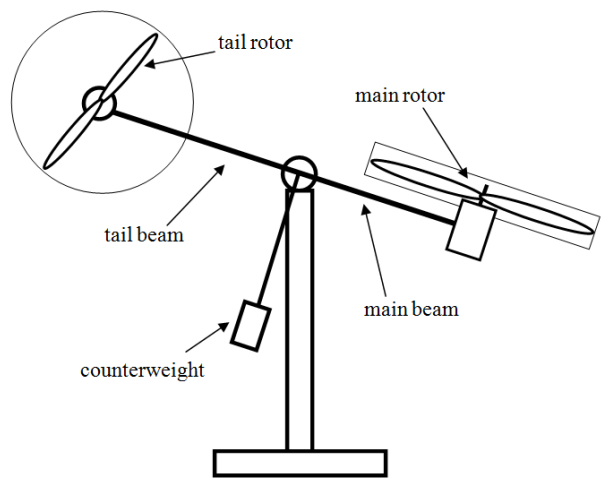

Figure 1. The model of TRMS

measured by tachogenerators coupled with the driving DC motors. Therefore, the control inputs are the supplied voltages of the DC-motors. A counterbalance arm with a weight at its end is fixed to the beam at the pivot. The main objective of this research is to control the outputs of the system, i.e., the pitch and azimuth angles.

Various control methods have been applied to TRMS. Gorczyca and Hajduk (Gorczyca \& Hajduk, 2004) designed the suboptimal and variable gain controller using the LQ methods for the TRMS. In Rahideh et al. (2007), a PD controller tuned with a genetic algorithm was used. To eliminate the inversion error, an adaptive neural network-based compensator was designed and added to the control system. Wen and $\mathrm{Lu}$ (2008) applied a PID-based robust deadbeat control technique to a TRMS, and they compared results with the system responses obtained from the individual PID controllers. In their work, the system was decoupled into two SISO systems, and the crossing couplings were considered as disturbances to each other. Also, they designed controllers for two SISO systems, and they could suppress the cross-coupling effects between the tail and the main rotors. In Juang et al. (2008), several classical and intelligent control techniques were applied to a TRMS for trajectory tracking control. The system equipped with the fuzzy controller had better performance than other controllers. In Tao et al. (2010), the TRMS was decoupled, and Takagi-Sugeno fuzzy model was used in detail. Based on this model of TRMS, parallel distributed fuzzy LQR controllers were presented, and the stability of the Takagi-Sugeno fuzzy TRMS model with these controllers was discussed. Juang et al. (2011) employed a conventional PID controller and a fuzzy compensator with a real-valued genetic algorithm for tuning parameters of the PID controller. Augmented PID with feedforward and feedback inverse-model control with particle swarm optimization was presented in (Toha \& Tokhi, 2011) and, the results were compared to the conventional PID controller based methods. A fault-tolerant control strategy based on a kind of virtual actuator approach using linear parameter varying techniques was presented in (de Oca et al., 2012). Jahed and Farrokhi (2013) designed two adaptive fuzzy con- trollers, and they applied these controllers to a TRMS in real-time. Moreover, based on the Lyapunov theory, they compared the experimental results of these controllers with the results obtained from the nonadaptive fuzzy and PID controllers. Zeghlache et al. (2014) implemented a type-2 fuzzy logic controller for the TRMS. They also compared the performance of this controller with the performance of the type- 1 fuzzy logic controller. Realtime implementation of a neuroadaptive observer-based robust backstepping controller for the TRMS and estimation of the unknown nonlinearities of the TRMS using Chebyshev neural network were investigated in (Pratap \& Purwar, 2014). Butt and Aschemann (2015) designed a integral sliding mode controller and they employed a discrete-time extended Kalman filter combined with a discrete-time implementation of the nonlinear control law to estimate unmeasurable states, uncertainties, and disturbance torques. Pandey et al. (2016) proposed a multiple model-based adaptive control with static statefeedback based on feedback linearization technique on the TRMS. Choudhary (2017) designed an $H^{\infty}$ optimal feedback controller enhanced with a mixed sensitivity method in the presence of cross-coupling effects, and he compared the transient performance of $H^{\infty}$ - controlled system with classical PID design. In Pandey et al. (2016), a robust PID controller based on the Kharitonov's stability theorem was designed and implemented on a TRMS. Designing and real-time implementation of a decentralized sliding mode controller for a TRMS with a sigmoid function in the sliding surface to remedy chattering problems were studied in (Faris et al., 2017). Precup et al. (2017) proposed two model-free sliding mode control system structures based on Lyapunov stability theory, and they compared with a model-free intelligent PI control system structure. A practically implementable model predictive controller design for a TRMS was presented in (Raghavan \& Thomas, 2017). Two proportional-integral-derivative controllers were employed in (Pandey et al., 2018) to control the main and tail rotors of TRMS, independently. Kharitonov's robust stability theorem was used to evaluate the ranges of the controller gains in the presence of structured uncertainties. Bacterial foraging optimization technique was also used to find the parameter value meeting desirable time domain specifications.

An accurate dynamic model of the system is required to develop effective control objectives, and this is a more powerful motive to design a controller that can control the system with cross-coupling and uncertainties, hence, the Quantitative Feedback Theory (QFT) is selected as one of the robust control methods.

Quantitative feedback theory, introduced by Horowitz in the early 1960s, is a frequency domain technique that emphasizes the use of feedback for achieving the desired system performance tolerances despite plant uncertainty and plant disturbances (Houpis \& Rasmussen, 1999). Utilizing the Nichols chart is the key tool for this technique, and it is used to achieve a robust design over 
the specified region of plant uncertainty. Desired timedomain responses are translated into frequency domain tolerances, which lead to bounds (or constraints) on the loop transmission function. The design process enables the designer to a trade-off between compensator complexity and system performance (Gharib \& Moavenian, 2016; Jahanpour et al., 2015; Moavenian et al., 2011; Moeinkhah, et al., 2014).

In this paper, to obtain the transfer functions, the equations of the electrical and mechanical part of TRMS are linearized. Then, the linear model was compared with the nonlinear one for each plane by a simulation approach. Also, using the QFT method, a controller is designed for motion in each plane. Furthermore, the robustness of the control strategy is illustrated by the time domain closedloop responses of vertical and horizontal motions, including the controller and pre-filter in the presence of uncertainties.

\section{Modeling the TRMS}

In this section, the mathematical model of TRMS introduced by Christensen et al. (2006) is briefly presented. The TRMS model is divided into a vertical and a horizontal plane. Also there are three sections, namely the DC-motors, the rotors, and the mechanics for each plane. The DC-motor has voltage as input and angular velocity as output. The rotor has an angular velocity as input and returns the aerodynamic torques. The inputs for the mechanics of the system are an aerodynamic force from the same plane, and an aerodynamic force and the angular velocity from the other plane (the cross-coupling between two planes). The output from the mechanical section is the pitch and the azimuth angles for the vertical and the horizontal planes, respectively.

\subsection{Model of DC-motors}

The electrical and mechanical parts of the DC-motor can be modeled as the following equations, respectively:

$$
\begin{aligned}
& u_{a}(t)=R_{a} i_{a}(t)+L_{a} \frac{d i_{a}(t)}{d t}+k \omega_{a}(t) ; \\
& J_{a} \dot{\omega}_{a}(t)=k i_{a}(t)-D_{a} \omega_{a}^{2}(t)-B_{a} \omega_{a}(t),
\end{aligned}
$$

where $u_{a}$ is the applied motor voltage, $R_{a}$ is the Ohmic resistance, $i_{a}$ is the armature current, $L_{a}$ is the inductance, $k$ is the motor constant, $\omega_{a}$ is the angular velocity of the armature, $J_{a}$ is the total inertia seen by the motor, $D_{a}$ is the motor drag friction constant; accordingly, $D_{a} \omega_{a}^{2}(t)$ is the torque due to drag friction. $B_{a}$ is the motor viscous friction constant; consequently, $B_{a} \omega_{a}(t)$ is the torque due to viscous friction.

To simplify the model of the DC motor system, the term $L_{a} \frac{d i_{a}(t)}{d t}$ can be ignored in equation (1). As a result, the system dynamics is not altered significantly by omitting the inductance of the motor because the time constant of the linear term $L_{a} \frac{d i_{a}(t)}{d t}$ is small compared to the mechanical part (Christensen et al., 2006).

Thus, equation (1) reduces to the following linear equation:

$$
u_{a}(t)=R_{a} i_{a}(t)+k \omega_{a}(t) .
$$

The nonlinear terms of the model are linearized using a first order Taylor approximation (Greenberg, 1998; Jeffrey, 2002).

$$
f(x) \approx f(\bar{x})+\left.\frac{d f}{d x}\right|_{x=\bar{x}}(x-\bar{x}) .
$$

That can be applied to a function of two variables (Greenberg, 1998; Jeffrey, 2002):

$$
f(x, y) \approx f(\bar{x}, \bar{y})+\left.\frac{\partial f}{\partial x}\right|_{(\bar{x}, \bar{y})}(x-\bar{x})+\left.\frac{\partial f}{\partial y}\right|_{(\bar{x}, \bar{y})}(y-\bar{y}) .
$$

Hence, the linear equations of electrical and mechanical parts of the DC-motor are obtained, respectively:

$$
\begin{aligned}
& \tilde{u}_{a}(t)=R_{a} \tilde{i}_{a}(t)+k \tilde{\omega}_{a}(t) ; \\
& J_{a} \dot{\tilde{\omega}}_{a}(t)=k \tilde{i}_{a}(t)-2 D_{a} \bar{\omega}_{a} \tilde{\omega}_{a}(t)-B_{a} \tilde{\omega}_{a}(t),
\end{aligned}
$$

where $\tilde{u}_{a}(t)=u_{a}(t)-\bar{u}_{a}, i_{a}(t)=i_{a}(t)-\bar{i}_{a}$ and $\tilde{\omega}_{a}(t)=\omega_{a}(t)-\bar{\omega}_{a}{ }^{1}$.

The working point values $\bar{i}_{a}$ and $\bar{u}_{a}$ are calculated by choosing $\bar{\omega}_{a}$ (working point value of $\omega_{a}$ ) and solving equation (3) and equation (2) in steady-state:

$$
\begin{aligned}
& \bar{u}_{a}=R_{a} \bar{i}_{a}+k \bar{\omega}_{a} ; \\
& 0=k \bar{i}_{a}-D_{a} \bar{\omega}_{a}^{2}-B_{a} \bar{\omega}_{a} .
\end{aligned}
$$

The following results can be obtained by applying Laplace transforming from the equations and :

$$
\begin{aligned}
& \tilde{I}_{a}(s)=\frac{\tilde{U}_{a}(s)-k \tilde{\Omega}_{a}(s)}{R_{a}} ; \\
& \tilde{\Omega}_{a}(s)=\frac{k}{J_{a} s+2 D_{a} \bar{\omega}_{a}+B_{a}} \tilde{I}_{a}(s),
\end{aligned}
$$

where $\tilde{I}_{a}(s)=I_{a}(s)-\bar{i}_{a}, \tilde{\Omega}_{a}(s)=\Omega_{a}(s)-\bar{\omega}_{a}$ and $\tilde{U}_{a}(s)=U_{a}(s)-\bar{u}_{a}$.

The closed-loop transfer function is obtained from the block diagram of the linear DC-motors (Figure 2):

$$
\frac{\tilde{\Omega}_{a}(s)}{\tilde{U}_{a}(s)}=\frac{k}{J_{a} R_{a} s+R_{a}\left(2 D_{a} \bar{\omega}_{a}+B_{a}\right)+k^{2}} .
$$

\footnotetext{
1 The signs " " and "-" at the top of variables are indicated the small signal and the working point values, respectively.
} 


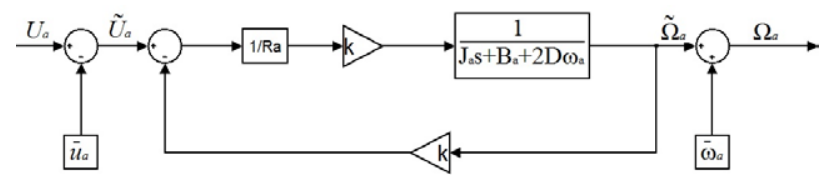

Figure 2. Block diagram of the linear DC motor model in the working point

\subsection{Aerodynamic torques}

The aerodynamic torques modeling using the theoretical methods would be too difficult. Using a black-box test is much simpler and is more likely to produce more accurate results. The thrust and drag torques for both rotors were determined in (Christensen et al., 2006) using black-box test method. The model of the aerodynamic torques is:

For the main rotor:

$\tau_{m}=a_{1} \omega_{m}^{2}(t)+a_{2} \omega_{m}(t)=57.5 \times 10^{-6} \omega_{m}^{2}+0.713 \times 10^{-3} \omega_{m} ;$

$\tau_{m d}=c_{1} \omega_{m}^{2}(t)+c_{2} \omega_{m}(t)=4.80 \times 10^{-6} \omega_{m}^{2}-9.35 \times 10^{-6} \omega_{m}$.

For the tail rotor:

$\tau_{t}=d_{1} \omega_{t}^{3}(t)+d_{2} \omega_{t}^{2}(t)+d_{3} \omega_{t}(t)=-59.2 \times 10^{-9} \omega_{t}^{3}+$

$2.65 \times 10^{-6} \omega_{t}^{2}-0.251 \times 10^{-3} \omega_{t}$;

$\tau_{t d}=b_{1} \omega_{t}^{3}(t)+b_{2} \omega_{t}^{2}(t)+b_{3} \omega_{t}(t)=5.49 \times 10^{-9} \omega_{t}^{3}-$

$0.206 \times 10^{-6} \omega_{t}^{2}+32.5 \times 10^{-6} \omega_{t}$.

In the above relations, $\omega_{m}$ is the angular velocity of the main rotor, $\omega_{t}$ is the angular velocity of the tail rotor, $\tau_{m}$ and $\tau_{t}$ are the thrust torques, $\tau_{m d}$ and $\tau_{t d}$ are the rotor drag torques from the main and the tail rotors, respectively.

\subsection{Modeling the mechanics}

In this subsection, the torques and the moment of inertia for motion in each plane are calculated. Then using the conservation law of angular momentum, the equations of motions are obtained. All the mass and length parameters used in this subsection are shown in Figure 3, and their numerical values are given in the Appendix.

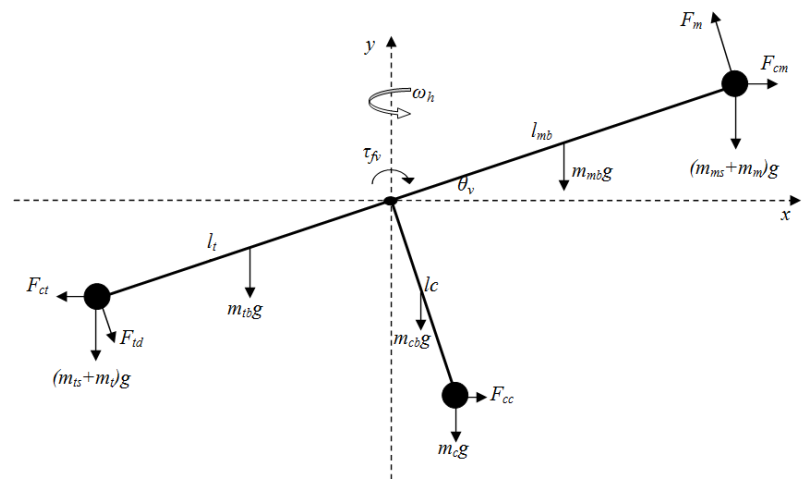

Figure 3. Total forces and torques in the vertical plane

\subsubsection{Torques in the vertical plane}

In the vertical plane, the torques are (Figure 3):

_ $\tau_{m}$ is the main rotor thrust torque which is results from the main rotor thrust force $F_{m}$

- $\tau_{t d}$ is the tail rotor drag torque which is results from the tail rotor drag force $F_{t d}$

- torque $\tau_{g}$ produced by the gravitational force

- torque $\tau_{c}$ produced by the centrifugal forces

- torque $\tau_{f v}$ produced by the friction force

Considering Figure 3, the net torque exerted by the gravitation on the TRMS is:

$$
\tau_{g}(t)=g\left((A-B) \cos \left(\theta_{v}(t)\right)-C \sin \left(\theta_{v}(t)\right)\right),
$$

where $\theta_{v}$ is the pitch angle, also:

$$
\begin{aligned}
& A=\left(\frac{m_{t b}}{2}+m_{t s}+m_{t}\right) l_{t b} \\
& B=\left(\frac{m_{m b}}{2}+m_{m s}+m_{m}\right) l_{m b} . \\
& C=\left(\frac{m_{c b}}{2}+m_{c}\right) l_{c}
\end{aligned}
$$

The total torque from the centrifugal forces $F_{c m}, F_{c t}$, and $F_{c c}$ is:

$$
\tau_{c}(t)=-\frac{1}{2} \omega_{h}^{2}(t)\left(A l_{t b}+B l_{m b}-C l_{c}\right) \sin \left(2 \theta_{v}(t)\right),
$$

where $\omega_{h}=\dot{\theta}_{h}$ in which $\theta_{h}$ is the azimuth angle.

The torque caused by the viscous friction is:

$$
\tau_{f v}(t)=-\omega_{v}(t) \mu_{v},
$$

where $\omega_{v}=\dot{\theta}_{v}$ and also $\mu_{v}$ is the viscous friction constant in the vertical plane.

From equations (17), (19) and (20) combined with equations (13) and (16), the net torque in the vertical plane is:

$$
\begin{aligned}
\tau_{v}(t)= & g\left((A-B) \cos \left(\theta_{v}(t)\right)-C \sin \left(\theta_{v}(t)\right)\right) \\
& -\frac{1}{2} \omega_{h}^{2}(t)\left(A l_{t b}+B l_{m b}-C l_{c}\right) \sin \left(2 \theta_{v}(t)\right) . \\
& -\omega_{v}(t) \mu_{v}+\tau_{m}\left(\omega_{m}(t)\right)+\tau_{t d}\left(\omega_{t}(t)\right)
\end{aligned}
$$

\subsubsection{Torques in the horizontal plane}

In the horizontal plane, the torques are (Figure 4):

- $\tau_{t}$ is the tail rotor thrust torque which is results from the tail rotor thrust force $F_{t}$

- $\tau_{m d}$ is the main rotor drag torque which is results

from the main rotor drag force $F_{m d}$

- torque $\tau_{f h}$ produced by the friction force

- torque $\tau_{k h}$ produced by the spring force

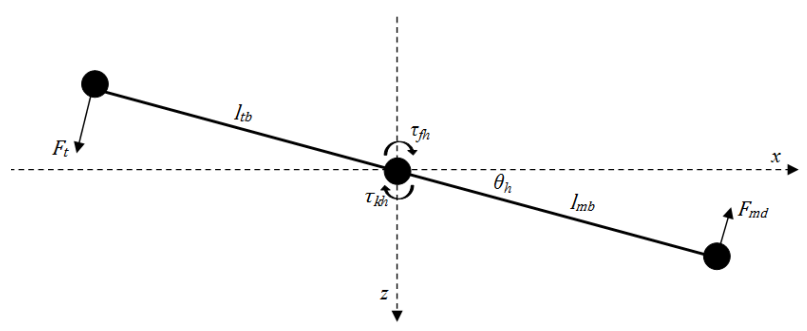

Figure 4. Total forces and torques in the horizontal plane 
The torque $\tau_{f h}$ produced by the friction force in the horizontal plane is:

$$
\tau_{f h}(t)=-\omega_{h}(t) \mu_{h},
$$

where $\mu_{h}$ is the viscous friction constant in the horizontal plane.

The spring torque arising from computer cables is:

$$
\tau_{k h}(t)=-\theta_{h}(t) k_{h},
$$

where $k_{h}$ is the spring constant in the horizontal plane.

From equations (22) and (23) combined with equations (14) and (15), the net torque in the horizontal plane is:

$\tau_{h}(t)=-\omega_{h}(t) \mu_{h}-\theta_{h}(t) k_{h}+\tau_{m d}\left(\omega_{m}(t)\right)+\tau_{t}\left(\omega_{t}(t)\right)$.

\subsubsection{Moment of inertia in the vertical plane}

Using the parallel axis theorem (Ginsberg, 2008; Harrison \& Nettleton, 1997; Meriam \& Kraige, 2002) and mere simplifications, the total moment inertia in the vertical plane is:

$$
\begin{aligned}
J_{v}= & m_{m} l_{m b}^{2}+\frac{1}{3} m_{m b} l_{m b}^{2}+m_{t} l_{t b}^{2}+\frac{1}{3} m_{t b} l_{t b}^{2}+m_{c} l_{c}^{2} \\
& +\frac{1}{3} m_{c b} l_{c}^{2}+\frac{1}{2} m_{m s} r_{m s}^{2}+m_{m s} l_{m b}^{2}+m_{t s} r_{t s}^{2}+m_{t s} l_{t b}^{2} .
\end{aligned}
$$

\subsubsection{Moment of inertia in the horizontal plane}

The moment of inertia of each part can be obtained by calculating the moment of inertia of each rigid body around an inclined axis (D'Souza \& Garg, 1984; Ginsberg, 2008; Harrison \& Nettleton, 1997; Meriam and Kraige, 2002) and the parallel-axis theorem along with some simplifying procedures. Hence, the total moment of inertia in the horizontal plane is:

$$
J_{h}(t)=D+E \cos ^{2}\left(\theta_{v}(t)\right)+F \sin ^{2}\left(\theta_{v}(t)\right)
$$

where:

$$
\begin{aligned}
& D=\frac{1}{2} m_{t s} r_{t s}^{2} \\
& E=m_{m} l_{m b}^{2}+\frac{1}{3} m_{m b} l_{m b}^{2}+m_{t} l_{t b}^{2}+\frac{1}{3} m_{t b} l_{t b}^{2}+m_{m s} r_{m s}^{2}+ \\
& m_{m s} l_{m b}^{2}+m_{t s} l_{t b}^{2} ; \\
& F=m_{c} l_{c}^{2}+\frac{1}{3} m_{c b} l_{c}^{2}+\frac{1}{2} m_{m s} r_{m s}^{2} .
\end{aligned}
$$

\subsection{Transfer function in the vertical plane}

The following statement is derived by considering conservation law of angular momentum (Ginsberg, 2008; Harrison \& Nettleton, 1997) for the TRMS:

$$
H_{v}(t)=H_{v, \text { ext }}(t)+H_{t}(t),
$$

where $H_{v \text {,ext }}$ is the angular momentum arising from external torque affecting the TRMS, $H_{t}$ is the angular momentum from the tail rotor circling and $H_{v}(t)$ is the resulting angular momentum.

Differentiating equation (28), then use of a derivative form of the angular impulse-momentum principle, i.e. $\dot{H}_{O}=\Sigma \mathrm{M}_{O}$ (Ginsberg, 2008) for $\dot{H}_{v, \text { ext }}$ and applying the theorem of angular momentum $H=J \omega$ for the remaining terms yields:

$$
\tau_{v}=J_{v} \ddot{\theta}_{v}(t)-J_{t r} \ddot{\theta}_{t},
$$

where $\ddot{\theta}_{v}$ is the angular acceleration from external torques in the vertical plane, $\ddot{\theta}_{t}$ is the angular acceleration of the tail rotor and $J_{t r}$ is the moment of inertia of the tail rotor blades.

To calculate the moment of inertia of the tail rotor blades $J_{t r}$, the rotor is approximated as a rectangular plate with the inertia of $\frac{1}{12} m\left(a^{2}+b^{2}\right)$ (Ginsberg, 2008; Meriam \& Kraige, 2002):

$$
J_{t r}=\frac{1}{12} m_{t r}\left(l_{t w}^{2}+l_{t l}^{2}\right)
$$

where $m_{t r}$ is the mass of tail rotor, $l_{t w}$ is the width of the tail rotor blade, and $l_{t l}$ is the length of the tail rotor blade.

Substituting equation (21) in equation (29), then, linearizing the obtained equation, the following transfer function for the motion in the vertical plane based on the angular velocity of the main and tail rotor is obtained (Christensen et al., 2006):

$$
\begin{gathered}
\tilde{\Theta}_{v}(s)=\frac{\left(2 a_{1} \bar{\omega}_{m}+a_{2}\right)}{J_{v} s^{2}+\mu_{v} s+g C} \tilde{\Omega}_{m}(s)+ \\
\frac{\left(J_{t r} s+3 b_{1} \bar{\omega}_{t}^{2}+2 b_{2} \bar{\omega}_{t}+b_{3}\right)}{J_{v} s^{2}+\mu_{v} s+g C} \tilde{\Omega}_{t}(s) .
\end{gathered}
$$

The numerical values of constant coefficients $a_{1}, a_{2}$, $b_{1}, b_{2}$ and $b_{3}$ in the above equation, were mentioned in section 1.2. Aerodynamic Torques.

\subsection{Transfer function in the horizontal plane}

The transfer function in the horizontal plane is determined similarly to the vertical one. As a general rule, the conservation of angular momentum states:

$$
H_{h}(t)=H_{h, \text { ext }}(t)+H_{m}(t) \cos \left(\theta_{v}\right),
$$

where $H_{h}$ is the resulting angular momentum. $H_{h, \text { ext }}$ is the angular momentum arising from the external torque affecting the TRMS, and $H_{m}$ is the angular momentum arising from the spinning main rotor. Substituting $H=J \omega$ in equation (32) and differentiating it, then, by use of Newton's Second Law, i.e., $\Sigma \mathrm{M}_{O}=J \ddot{\theta}$ and applying it for $\ddot{\theta}_{h}(t)=\dot{\omega}_{h}(t)=\frac{\tau_{h}}{J_{h}(t)}$, the following relation is
stated:

$\tau_{h}=J_{h}(t) \dot{\omega}_{h}(t)-$
$J_{m r}\left[\dot{\omega}_{m}(t) \cos \left(\theta_{v}(t)\right)-\omega_{m}(t) \dot{\theta}_{v}(t) \sin \left(\theta_{v}(t)\right)-\frac{J_{h}(t) \omega_{m}(t) \cos \left(\theta_{v}(t)\right)}{J_{h}(t)}\right]$.

Moment of inertia of the main rotor blades $J_{m r}$ is also calculated similarly to which performed for $J_{t r}$ :

$$
J_{m r}=\frac{1}{12} m_{m r}\left(l_{m w}^{2}+l_{m l}^{2}\right),
$$

where $m_{m r}$ is the mass of the main rotor, $l_{m w}$ is the width of the main rotor blade, and $l_{m l}$ is the length of the main rotor blade. 
Differentiating from equation (26) for $\dot{J}_{h}(t)$ and substituting equation (24) in equation (33), then linearizing the obtained equation, the following transfer function for the motion in the horizontal plane based on the angular velocity of the main and tail rotor is obtained (Christensen et al., 2006):

$$
\begin{aligned}
& \tilde{\Theta}_{h}(s)=\frac{3 d_{1} \bar{\omega}_{t}^{2}+2 d_{2} \bar{\omega}_{t}+d_{3}}{(D+E) s^{2}+\mu_{h} s+k_{h}} \tilde{\Omega}_{t}(s)+ \\
& \frac{J_{m r} s+2 c_{1} \bar{\omega}_{m}+c_{2}}{(D+E) s^{2}+\mu_{h} s+k_{h}} \tilde{\Omega}_{m}(s) .
\end{aligned}
$$

The numerical values of constant coefficients $c_{1}, c_{2}, d_{1}$, $d_{2}$ and $d_{3}$ in the above equation, were mentioned in section 1.2. Aerodynamic Torques.

\section{Simulation results without controllers}

The working point is defined when the main beam is located horizontally, i.e., $\theta_{v}=0$ and $\theta_{h}=0$. In (Christensen et al., 2006), $\bar{\omega}_{a}$ has been obtained experimentally for both rotors: $\bar{\omega}_{m}=54 \mathrm{rad} / \mathrm{s}$ and $\bar{\omega}_{t}=-44 \mathrm{rad} / \mathrm{s}$.

The values of $\bar{i}_{a t}{ }^{2}, \bar{u}_{a t}, \bar{i}_{a m}$ and $\bar{u}_{a m}$ can be obtained by solving the steady-state equations and :

$$
\begin{array}{ll}
\bar{i}_{a t}=\frac{B_{a t} \bar{\omega}_{t}}{k_{t}} & =-0.125 \mathrm{~A} \\
\bar{u}_{a t}=R_{a t} \bar{i}_{a t}+k_{m t} \bar{\omega}_{t} & =-4.89 \mathrm{~V} \\
\bar{i}_{a m}=\frac{D_{a m} \bar{\omega}_{m}^{2}+B_{a m} \bar{\omega}_{m}}{k_{m}} & =1.15 \mathrm{~A} \\
\bar{u}_{a m}=R_{a m} \bar{i}_{a m}+k_{m} \bar{\omega}_{m} & =11.9 \mathrm{~V} .
\end{array}
$$

By inserting the numerical values from Appendix into equation (12), the following transfer functions for the tail and main motor are obtained, respectively:

$$
\frac{\Omega_{t}(s)}{\tilde{U}_{a t}(s)}=\frac{k_{t}}{J_{a t} R_{a t} s+R_{a t} B_{a t}+k_{t}^{2}}=\frac{0.0883}{0.00410 s+0.00980} ;
$$

$\frac{\tilde{\Omega}_{m}(s)}{\tilde{U}_{a m}(s)}=\frac{k_{m}}{J_{a m} R_{a m} s+R_{a m}\left(2 D_{a m} \bar{\omega}_{m}+B_{a m}\right)+k_{m}^{2}}=$

$$
\frac{0.0748}{0.0188 s+0.0249} \text {. }
$$

Besides, by inserting the numerical values from Appendix into equations (31) and (35), the following transfer functions for the motions in the vertical and horizontal planes based on the angular velocity of the main and tail rotors are obtained, respectively:

$$
\begin{aligned}
& \tilde{\Theta}_{v}(s)=\frac{0.00692}{0.0673 s^{2}+0.0021 s+0.288} \tilde{\Omega}_{m}(s)+ \\
& \frac{43.6 s+82.5}{0.0673 s^{2}+0.0021 s+0.288} \times 10^{-6} \tilde{\Omega}_{t}(s) ;
\end{aligned}
$$

2 The index " $\mathrm{m}$ " and " $\mathrm{t}$ " are referred to the main and tail rotor, respectively.

$$
\begin{aligned}
& \tilde{\Theta}_{h}(s)=\frac{0.362 \times 10^{-3}}{0.0642 s^{2}+0.0107 s+0.0093} \tilde{\Omega}_{t}(s) . \\
& +\frac{0.279 s+0.509}{0.0642 s^{2}+0.0107 s+0.0093} \times 10^{-3} \tilde{\Omega}_{m}(s) .
\end{aligned}
$$

The simulation for the nonlinear and linear model has been performed with Simulink in MATLAB'. The input signals for two DC-motors and motors responses to input signals are shown in Figure 5 and Figure 6, respectively.

As can be seen in Figure 6, for the main rotor model, the linear model response is approximately identical to the nonlinear model response. The two responses overlap at the working point exactly. However, for the tail rotor model, as we expected, the linearized model response curve falls precisely on the nonlinear model response curve, which is due to neglecting the drag friction coefficient $D_{a}$ for the tail rotor model in equation (2). The reason for ignoring this coefficient is given in the footnote of Appendix Table.
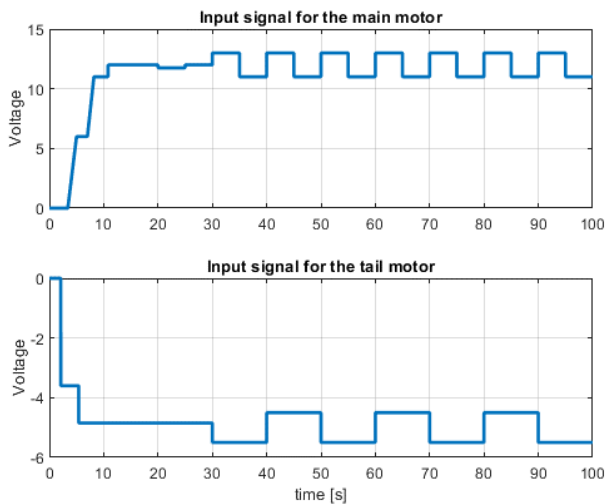

Figure 5. Input signals for DC-motors
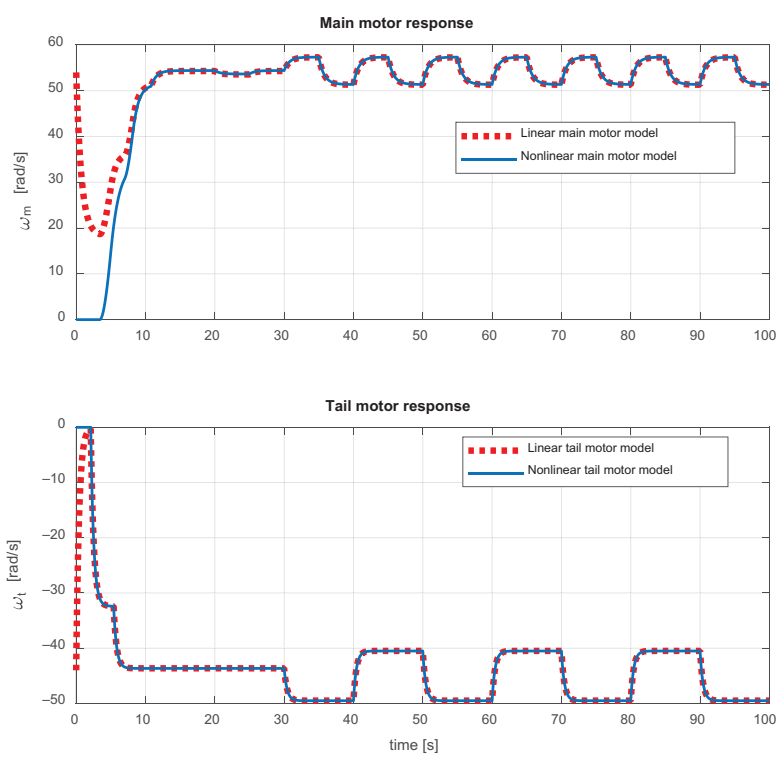

Figure 6. Motors responses 

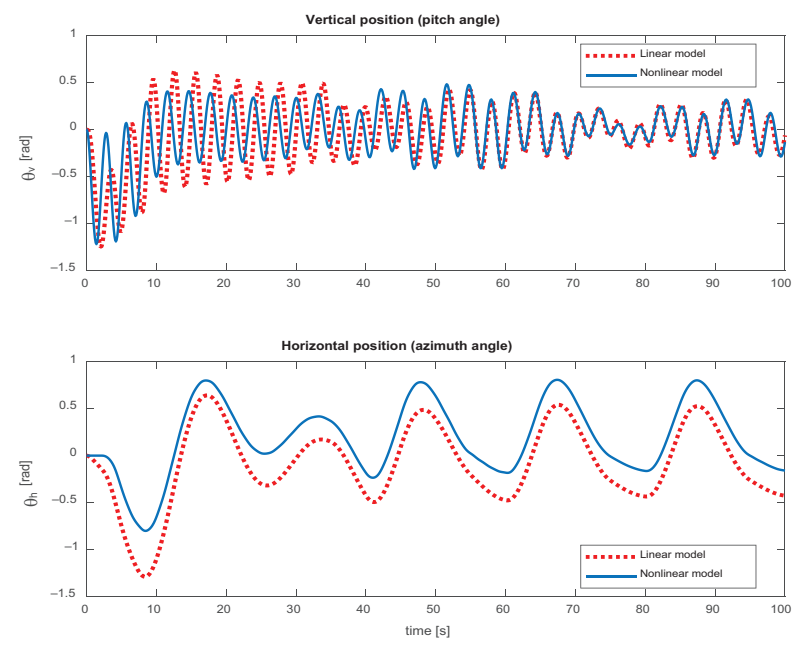

Figure 7. Final responses

By giving the input signals (Figure 5) to both the linear and nonlinear models of the device, the final output of the linear model and the nonlinear model are compared in Figure 7. Because of using the first-order Taylor approximation for obtaining the linear model and some simplifications, the difference between the curves of the linear model and the nonlinear model is seen in Figure 7. For the horizontal motion, this difference is more pronounced due to the presence of $\theta_{v}$ in the moment of inertia in the horizontal plane as well as the model complexity in comparison with the vertical plane model.

Despite some differences, the final outputs of the models shown in Figure 7 represent that the linear modeling of the electrical and mechanical parts is accurate adequately.

\section{Controller design and simulation}

The purpose of this work is to design a compensator $G$ and a pre-filter $\mathrm{F}$ for two-degree of freedom system shown in Figure 8 to achieve a desired robust design over a specified region of plant uncertainty. In this paper, consider an uncertain plant $\mathrm{p}(\mathrm{s})$ is belonging to a set of possible plants $p(s, \alpha)$, i.e. $p(s) \in\{p(s, \alpha) ; \alpha \in p\}$, where $\alpha$ is the vector of uncertain parameters for uncertainty structured of p(s) (Amiri-M et al., 2009; Gharib et al., 2010; Gharib \& Daneshvar, 2019; Gharib et al., 2011; Gharib \& Moavenian, 2016; Horowitz, 1992; Jahanpour et al., 2015).

This section uses the QFT method (Amiri-M et al., 2009; Gharib \& Moavenian, 2016; Jahanpour et al., 2015; Nataraj, 2002) to design a controller for each plane of the TRMS. The objectives of this section are to synthesize suitable controllers and pre-filters such that:

1. the closed-loop system is stable;

2. system tracks its desired input.

The stability margin is defined as:

$$
\left|\frac{P(j \omega) G(j \omega)}{1+P(j \omega) G(j \omega)}\right|<1.1 .
$$

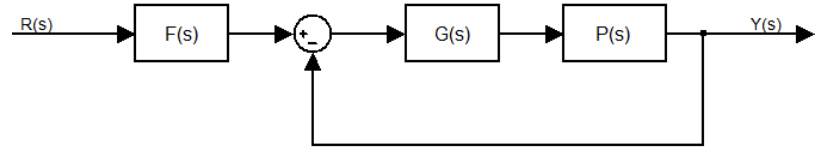

Figure 8. Structure of a two-degree of freedom system

The tracking specifications for closed-loop performance are given in the form of upper and lower bounds in the frequency domain (an overshoot of less than 10 percent and the settling time $=10 \mathrm{~s}$ ). Usually, based on the simple second-order models to represent appropriate under- and over-damped closed-loop system, the following is stated:

$$
a(j \omega)<F(j \omega) \frac{P(j \omega) G(j \omega)}{1+P(j \omega) G(j \omega)}<b(j \omega),
$$

$a(j \omega)$ and $b(j \omega)$ are the lower and upper bounds, respectively.

At the first step of controller design, a set of working frequencies is selected. Since the plant uncertainty must be defined (Template Generation), thus, the boundary of the plant templates should be computed. The area on the Nichols chart, which defines a graphical representation of the uncertainties at each frequency, is called the plant template. These templates, related to the selected working frequencies, are then used together with the performance specifications to define regions (or the so-called bounds) in the frequency domain where the open-loop frequency response must lie to satisfy the performance and stability specifications. The stability bounds are calculated using these templates and the phase margin. The tracking performance bounds are derived using the templates and the upper and lower limits on the frequency-domain responses. The upper limit of the disturbance bounds is derived based on the disturbance rejection specifications. Some other performance bounds, such as control action bounds, noise rejections bounds, etc., are also derived based on the templates and the corresponding performance limits. Finally, the composite performance bounds are constructed by taking the most restrictive regions of all considered performance bounds.

To summarize, the controller designing steps are just illustrated for vertical motion. Figure 9 shows plant templates for the vertical plane on the Nichols chart. Robust margin bounds are depicted in Figure 10. Robust tracking bounds are also shown in Figure 11. Besides, the intersection of bounds is shown in Figure 12.

The controller design is undertaken on the Nichols chart, considering the frequency constraints and the nominal loop $L_{0}(s)$ of the system. At this point, the designer begins to introduce controller functions $(G(s))$ and tune their parameters until the best possible controller is reached without violation of the frequency constraints. This process is called Loop Shaping.

One can design the controller using the QFT toolbox in MATLAB ${ }^{\circ}$ so that the open-loop transfer function exactly lies on its robust performance bounds and does not 


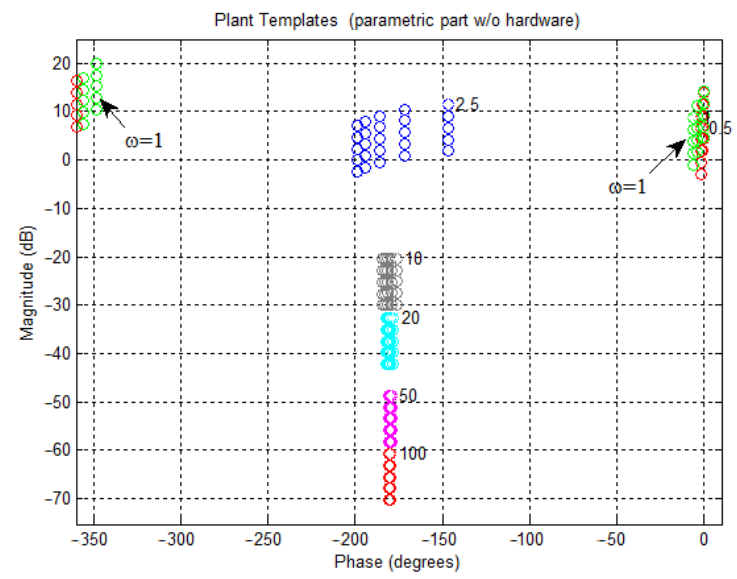

Figure 9. The boundary of the plant template for the vertical plane

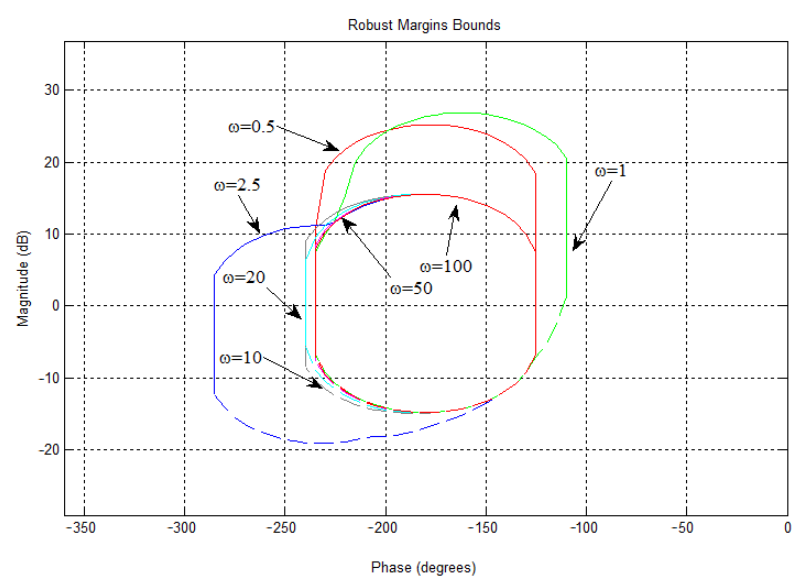

Figure 10. The robust margin for vertical plane

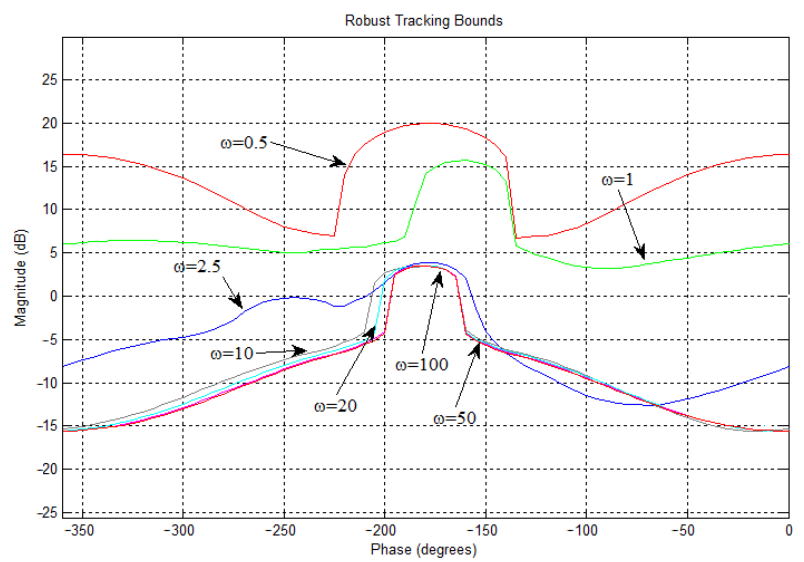

Figure 11. The robust tracking for vertical plane

penetrate the U-contour at all frequency values $\left(\omega_{i}\right)$. The experience of the designer is an essential factor in finding satisfactory controllers that not only comply with the frequency restrictions but also provide the possible realization, complexity, and quality.

Finally, the QFT design may be completed with a pre-filter $(\mathrm{F}(\mathrm{s}))$ design when it is required. In the case of tracking conditions, shaping on the Bode diagram may be used. Post design analysis is then performed to ensure the

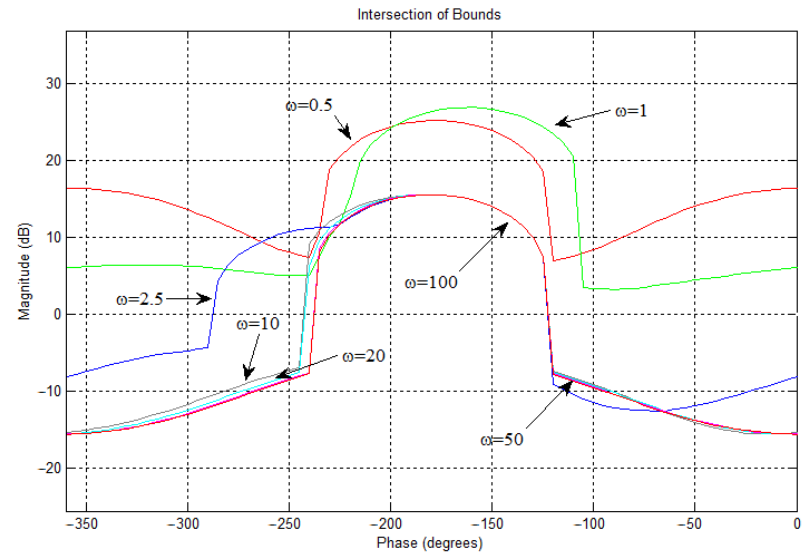

Figure 12. The intersection of bounds for the vertical plane

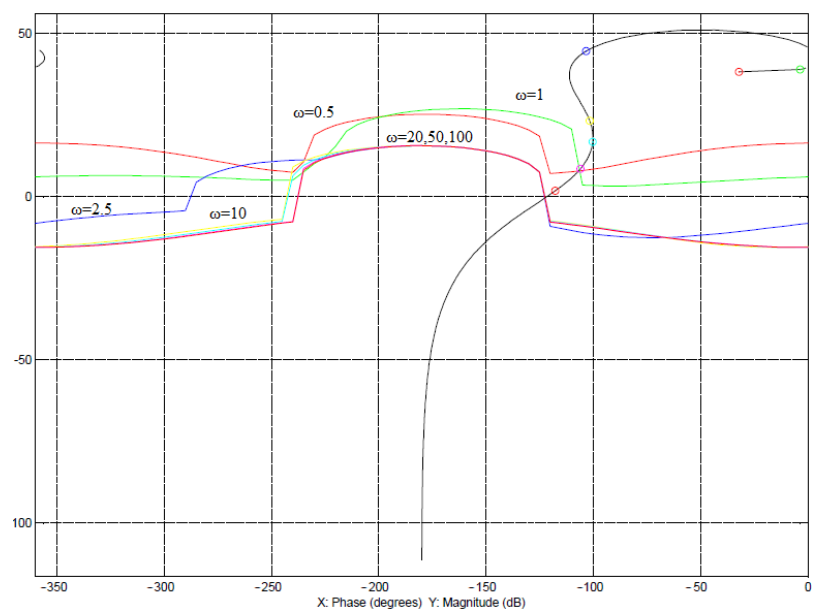

Figure 13. Loop-shaping in Nichols chart for the vertical plane

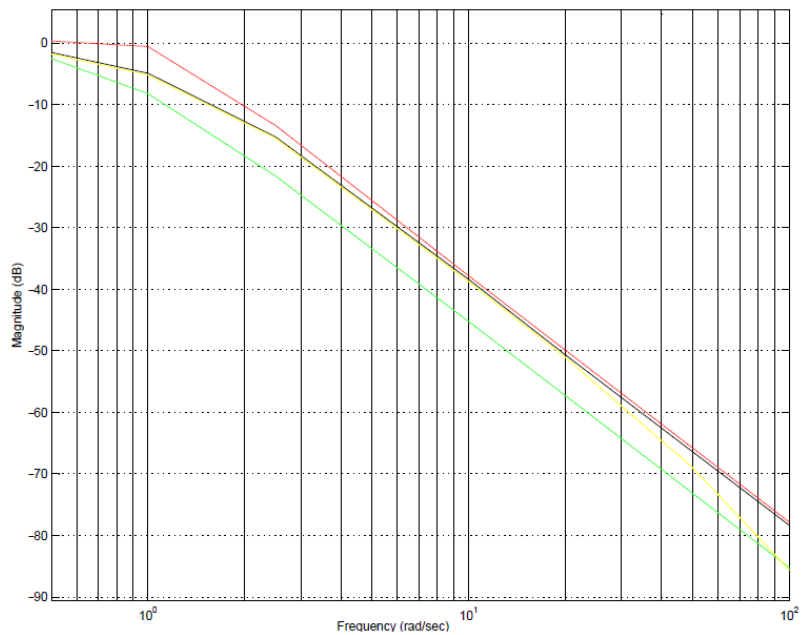

Figure 14. Pre-filter shaping for the vertical plane

system response is satisfactory according to the problem requirements.

Loop and pre-filter shaping of the open-loop transfer function for the vertical motion are presented in Figure 13 and Figure 14, respectively. 
The respected controller and pre-filter for the vertical $\left(G_{p} F_{v}\right)$ and the horizontal $\left(G_{h}, F_{h}\right)$ motions are found as follows:

$$
\begin{aligned}
& G_{v}(s)=\frac{2.94 s^{2}+6.44 s+2.6}{s^{2}+198 s} \times 10^{3} \\
& G_{h}(s)=\frac{3.15 s^{3}+37 s^{2}+48.7 s+25.1}{s^{3}+849 s^{2}+18000 s+1520} \times 10^{4} \\
& F_{v}(s)=\frac{1.14 s+3.7}{s^{3}+4.5 s^{2}+7.67 s+3.7} \\
& F_{h}(s)=\frac{0.0519 s+1.18}{s^{2}+2.44 s+1.18}
\end{aligned}
$$

Figure 15 illustrates robust stability in the frequency domain for the vertical plane.

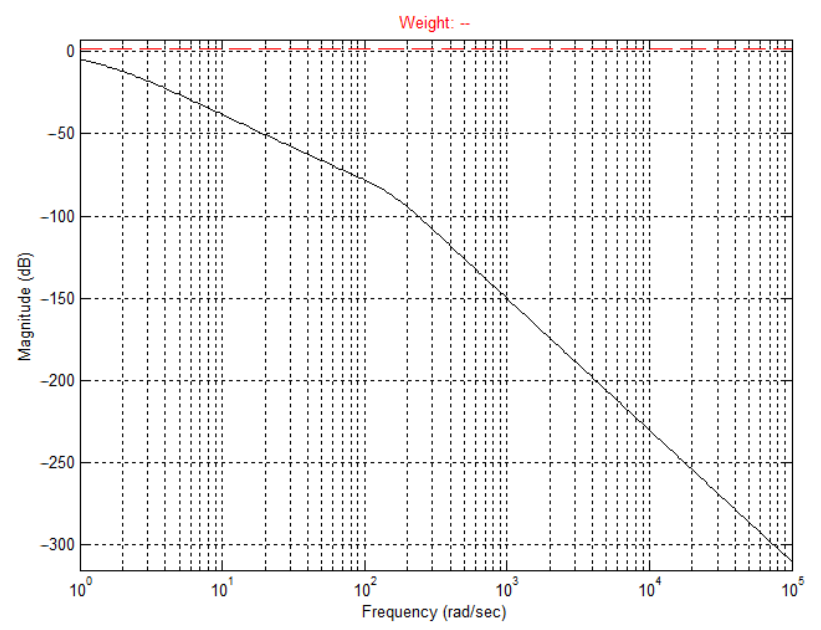

Figure 15. Robust stability in the frequency domain for the vertical plane

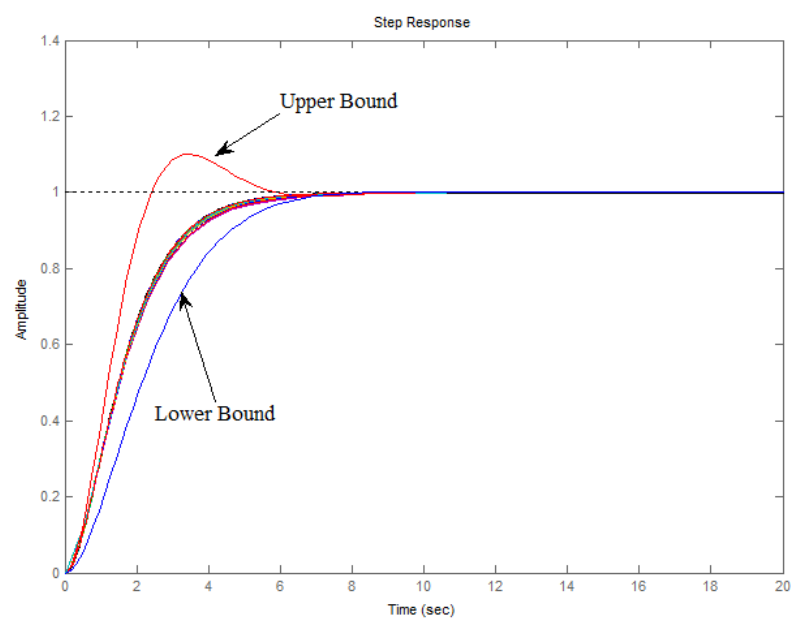

Figure 16. Step response of the system for vertical motion

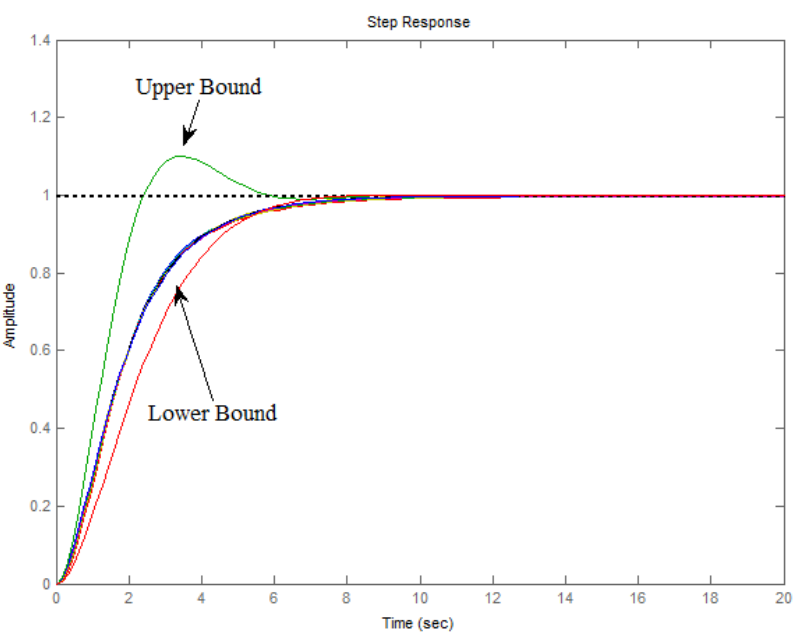

Figure 17. Step response of the system for horizontal motion

The time-domain closed-loop responses of vertical and horizontal motions, including the controller and pre-filter, are shown in Figure 16 and Figure 17, respectively. Regarding the simulation results, it can be seen that the QFT control approach not only overcomes the uncertainties of the TRMS but also can satisfy robust stability and tracking specifications of the system.

\section{Conclusions}

In this paper, the dynamic model of TRMS for motion in both vertical and horizontal planes has been introduced. For simplifying the modeling procedure, the TRMS model has been divided into the vertical and the horizontal planes and three sections for each plane. These sections are the DC-motors, the rotors, and the mechanics. To obtain the transfer functions, the equations of the electrical and mechanical part of TRMS were linearized. Then, by simulation, the linear model was compared with the nonlinear one for each plane, which indicates that:

1. The linear model of the electrical part is fitted with the nonlinear model close to the working point for each plane, and the tail motor two curves are fitted tightly even far from the working point. The reason arises from disregarding the second-order term for the tail rotor.

2. The linear model of the overall system is fitted with the nonlinear one with some differences for each plane. One reason for this difference arises from the use of first-order Taylor approximation for obtaining the linear model and some simplifications.

3. For the horizontal motion, the difference between the two curves is more dominant. One reason could be the existence of $\theta_{v}$ in the moment of inertia in the horizontal plane, which differs by changing it in the vertical angle. The other reason is that the model of the horizontal motion is more complicated than the model of the vertical motion. 
With a short glance at Equation (39), it can be intensely seen that the order of numerator in the second term is smaller than the order of numerator in the first term. There is not a crucial problem by ignoring the second term, and eliminate the cross-coupling effect in the analysis of motion in the vertical plane.

At the end part of this paper, utilizing the QFT approaches as a famous and applicable designing robust controllers for the TRMS has been proposed. The simulation results for tracking problem in vertical and horizontal planes indicate successful implementation of robust controller design for TRMS motion, despite there are uncertainties.

Finally, by comparing the theoretical results of this article with the experimental results, we can state the accuracy of the proposed simulation and controllers.

\section{References}

Amiri-M, A.-A., Gharib, M., Moavenian, M., \& Torabiz, K. (2009). Modelling and control of a SCARA robot using quantitative feedback theory. Proceedings of the Institution of Mechanical Engineers, Part I: Journal of Systems and Control Engineering, 223(7), 919-928. https://doi.org/10.1243/09596518JSCE733

Butt, S. S., \& Aschemann, H. (2015). Multi-variable integral sliding mode control of a two degrees of freedom helicopter. IFAC-PapersOnLine, 48(1), 802-807. https://doi.org/10.1016/j.ifacol.2015.05.129

Choudhary, S. K. (2017). Optimal feedback control of a twin rotor MIMO system. International Journal of Modelling and Simulation, 37(1), 46-53.

https://doi.org/10.1080/02286203.2016.1233008

Christensen, R., Fogh, N., Hansen, R. H., Jensen, M. S., Larsen, S., \& Paramanathan, A. (2006). Modelling and control of a twinrotor MIMO System. Report of Department of Control Engineering Institute of Electronic Systems of Aalborg University.

D’Souza, A. F., \& Garg, V. K. (1984). Advanced dynamics: modeling and analysis. Prentice-Hall, Inc.

de Oca, S., Puig, V., Witczak, M., \& Dziekan, Ł. (2012). Faulttolerant control strategy for actuator faults using LPV techniques: Application to a two degree of freedom helicopter. International Journal of Applied Mathematics and Computer Science, 22(1), 161-171.

https://doi.org/10.2478/v10006-012-0012-y

Faris, F., Moussaoui, A., Djamel, B., \& Mohammed, T. (2017). Design and real-time implementation of a decentralized sliding mode controller for twin rotor multi-input multi-output system. Proceedings of the Institution of Mechanical Engineers, Part I: Journal of Systems and Control Engineering, 231(1), 3-13. https://doi.org/10.1177/0959651816680457

Feedback Instruments Ltd. (1998). Twin rotor MIMO System Advanced Teaching Manual 1 (33-007-4M5 User Manual). East Sussex, UK.

Feedback Instruments Ltd. (2006). Twin rotor MIMO System (33$949 S$ User Manual). East Sussex, UK.

Gharib, M. R., Dabzadeh, I., Mousavi, S., \& Kamelian, S. (2010). Robust controller design with QFT and sliding mode for boiler pressure. Proceedings of the 2010 International Conference on Modelling, Identification and Control (pp. 412-417). Okayama.

Gharib, M. R., \& Daneshvar, A. (2019). Quantitative-fuzzy controller design for multivariable systems with uncertainty. In- ternational Journal of Control, Automation and Systems, 17(6), 1515-1523. https://doi.org/10.1007/s12555-018-0567-y

Gharib, M. R., Kamelian, S., Seyyed Mousavi, S. A., \& Dabzadeh, I. (2011). Modelling and multivariable robust controller for a power plant. International Journal of Advanced Mechatronic Systems, 3(2), 119-128.

https://doi.org/10.1504/IJAMECHS.2011.040683

Gharib, M. R., \& Moavenian, M. (2016). Full dynamics and control of a quadrotor using quantitative feedback theory. International Journal of Numerical Modelling: Electronic Networks, Devices and Fields, 29(3), 501-519.

https://doi.org/10.1002/jnm.2101

Ginsberg, J. (2008). Engineering dynamics. Cambridge University Press.

Gorczyca, P., \& Hajduk, K. (2004). Tracking control algorithms for a laboratory aerodynamical system. International Journal of Applied Mathematics and Computer Sciense, 14(4), 469-475.

Greenberg, M. D. (1998). Advanced engineering mathematics. Prentice Hall.

Harrison, H. R., \& Nettleton, T. (1997). Advanced engineering dynamics. Arnold.

Horowitz, I. M. (1992). Quantitative Feedback Design Theory (QFT), vol. 1. QFT Publications.

Houpis, C. H., \& Rasmussen, S. J. (1999). Quantitative feedback theory: fundamentals and applications. Marcel Dekker.

Jahanpour, J., Honari-Torshizi, M., \& Gharib, M. R. (2015). VCNC contour following tasks using robust QFT controller. Iranian Journal of Science and Technology Transactions of Mechanical Engineering, 39(M1), 131-145.

Jahed, M., \& Farrokhi, M. (2013). Robust adaptive fuzzy control of twin rotor MIMO system. Soft Computing, 17(10), 18471860. https://doi.org/10.1007/s00500-013-1026-6

Jeffrey, A. (2002). Advanced engineering mathematics. Harcourt Academic Press.

Juang, J.-G., Lin, R.-W., \& Liu, W.-K. (2008). Comparison of classical control and intelligent control for a MIMO system. Applied Mathematics and Computation, 205(2), 778-791. https://doi.org/10.1016/j.amc.2008.05.061

Juang, J.-G., Liu, W.-K., \& Lin, R.-W. (2011). A hybrid intelligent controller for a twin rotor MIMO system and its hardware implementation. ISA Transactions, 50(4), 609-619.

https://doi.org/10.1016/j.isatra.2011.06.006

Meriam, J. L., \& Kraige, L. G. (2002). Engineering mechanics: Dynamics. John Wiley and Sons Inc.

Moavenian, M., Gharib, M., Daneshvar, A., \& Alimardani, S. (2011). Control of human hand considering uncertainties [Conference presentation]. The 2011 International Conference on Advanced Mechatronic Systems (pp. 17-22). Zhengzhou.

Moeinkhah, H., Akbarzadeh, A., \& Rezaeepazhand, J. (2014). Design of a robust quantitative feedback theory position controller for an ionic polymer metal composite actuator using an analytical dynamic model. Journal of Intelligent Material Systems and Structures, 25(15), 1965-1977.

https://doi.org/10.1177/1045389X13512906

Nataraj, P. S. (2002). Computation of QFT bounds for robust tracking specifications. Automatica, 38(2), 327-334. https://doi.org/10.1016/S0005-1098(01)00203-5

Oktay, T., Konar, M., Soylak, M., Sal, F., Onay, M., \& Kizilkaya, O. (2016). Increasing performance of autopilot guided small unmanned helicopter. International Journal of Mechanical and Mechatronics Engineering, 10(1), 133-139.

Oktay, T., \& Sal, F. (2015). Helicopter control energy reduction using moving horizontal tail. The Scientific World Journal, 2015. https://doi.org/10.1155/2015/523914 
Oktay, T., \& Sal, F. (2016). Combined passive and active helicopter main rotor morphing for helicopter energy save. Journal of the Brazilian Society of Mechanical Sciences and Engineering, 38(6), 1511-1525. https://doi.org/10.1007/s40430-015-0401-X

Pandey, S. K., Dey, J., \& Banerjee, S. (2016). Design and real-time implementation of robust PID controller for Twin Rotor MIMO System (TRMS) based on Kharitonov's theorem [Conference presentation]. 2016 IEEE 1st International Conference on Power Electronics, Intelligent Control and Energy Systems (ICPEICES) (pp. 1-6). Delhi. https://doi.org/10.1109/ICPEICES.2016.7853106

Pandey, S. K., Dey, J., \& Banerjee, S. (2018). Design of robust proportional-integral-derivative controller for generalized decoupled twin rotor multi-input-multi-output system with actuator non-linearity. Proceedings of the Institution of $\mathrm{Me}$ chanical Engineers, Part I: Journal of Systems and Control Engineering, 232(8), 971-982.

https://doi.org/10.1177/0959651818771487

Pandey, V. K., Kar, I., \& Mahanta, C. (2016). Control of twinrotor MIMO system using multiple models with second level adaptation. IFAC-PapersOnLine, 49(1), 676-681. https://doi.org/10.1016/j.ifacol.2016.03.134

Pratap, B., \& Purwar, S. (2014). Real-time implementation of neuro adaptive observer-based robust backstepping controller for twin rotor control system. Journal of Control, Automation and Electrical Systems, 25(2), 137-150.

https://doi.org/10.1007/s40313-013-0098-y

Precup, R.-E., Radac, M.-B., Roman, R.-C., \& Petriu, E. M. (2017). Model-free sliding mode control of nonlinear systems: Algorithms and experiments. Information Sciences, 381, 176-192. https://doi.org/10.1016/j.ins.2016.11.026
Raghavan, R., \& Thomas, S. (2017). Practically Implementable Model Predictive Controller for a Twin rotor Multi-Input Multi-Output System. Journal of Control, Automation and Electrical Systems, 28(3), 358-370.

https://doi.org/10.1007/s40313-017-0311-5

Rahideh, A., Shaheed, H., \& Bajodah, A. (2007). Adaptive nonlinear model inversion control of a twin rotor multi-input multi-output system using artificial intelligence. Proceedings of the Institution of Mechanical Engineers, Part G: Journal of Aerospace Engineering, 221(3), 343-351.

https://doi.org/10.1243/09544100JAERO155

Rahideh, A., Shaheed, M., \& Huijberts, H. (2008). Dynamic modelling of a TRMS using analytical and empirical approaches. Control Engineering Practice, 16(3), 241-259. https://doi.org/10.1016/j.conengprac.2007.04.008

Tao, C.-W., Taur, J.-S., \& Chen, Y. (2010). Design of a parallel distributed fuzzy LQR controller for the twin rotor multiinput multi-output system. Fuzzy Sets and Systems, 161(15), 2081-2103. https://doi.org/10.1016/j.fss.2009.12.007

Toha, S. F., \& Tokhi, M. O. (2011). PID and inverse-model-based control of a twin rotor system. Robotica, 29(6), 929-938. https://doi.org/10.1017/S0263574711000154

Vilchis, J. A., Brogliato, B., Dzul, A., \& Lozano, R. (2003). Nonlinear modelling and control of helicopters. Automatica, 39(9), 1583-1596. https://doi.org/10.1016/S0005-1098(03)00168-7

Wen, P., \& Lu, T.-W. (2008). Decoupling control of a twin rotor MIMO system using robust deadbeat control technique. IET control theory applications, 2(11), 999-1007. https://doi.org/10.1049/iet-cta:20070335

Zeghlache, S., Kara, K., \& Saigaa, D. (2014). Type-2 fuzzy logic control of a 2-DOF helicopter (TRMS system). Central European Journal of Engineering, 4(3), 303-315.

\section{Appendix}

\section{Nomenclature of the TRMS}

This Appendix contains symbols with numerical values about the TRMS that have been used in this paper (Christensen et al., 2006; Feedback Instruments Ltd, 1998).

\begin{tabular}{|c|l|c|c|}
\hline Symbol & \multicolumn{1}{|c|}{ Description } & Value & Unit \\
\hline$l_{c}=l_{c b}$ & Length of counterweight beam & 0.255 & $\mathrm{~m}$ \\
\hline$l_{m b}$ & Length of the main beam & 0.24 & $\mathrm{~m}$ \\
\hline$l_{t b}$ & Length of tail beam & 0.25 & $\mathrm{~m}$ \\
\hline$l_{m w}$ & The width of the main rotor blade & 0.026 & $\mathrm{~m}$ \\
\hline$l_{m l}$ & Length of the main rotor blade & 0.281 & $\mathrm{~m}$ \\
\hline$l_{t w}$ & The width of the tail rotor blade & 0.017 & $\mathrm{~m}$ \\
\hline$l_{t l}$ & Length of the tail rotor blade & 0.180 & $\mathrm{~m}$ \\
\hline$r_{m s}$ & The radius of the main shield & 0.155 & $\mathrm{~m}$ \\
\hline$r_{t s}$ & The radius of the tail shield & 0.100 & $\mathrm{~m}$ \\
\hline$m_{c}$ & Mass of counterweight & 0.068 & $\mathrm{~kg}$ \\
\hline$m_{c b}$ & Mass of counterweight beam & 0.022 & $\mathrm{~kg}$ \\
\hline$m_{m b}$ & Mass of the main beam & 0.0145 & $\mathrm{~kg}$ \\
\hline$m_{t b}$ & Mass of tail beam & 0.0155 & $\mathrm{~kg}$ \\
\hline$m_{m}$ & Mass of main DC-motor with the main rotor & 0.228 & $\mathrm{~kg}$ \\
\hline
\end{tabular}




\begin{tabular}{|c|l|c|c|}
\hline Symbol & \multicolumn{1}{|c|}{ Description } & Value & Unit \\
\hline$m_{t}$ & Mass of tail DC-motor with the tail rotor & 0.206 & $\mathrm{~kg}$ \\
\hline$m_{m s}$ & Mass of main shield & 0.225 & $\mathrm{~kg}$ \\
\hline$m_{t s}$ & Mass of tail shield & 0.165 & $\mathrm{~kg}$ \\
\hline$m_{m r}$ & Mass of the main rotor & 0.042 & $\mathrm{~kg}$ \\
\hline$m_{t r}$ & Mass of tail rotor & 0.016 & $\mathrm{~kg}$ \\
\hline$\mu_{v}$ & Viscous friction constant in the vertical plane & 0.0021 & $\mathrm{kgm}^{2} / \mathrm{s}$ \\
\hline$\mu_{h}$ & Viscous friction constant in the horizontal plane & 0.0107 & $\mathrm{kgm}^{2} / \mathrm{s}$ \\
\hline$k_{h}$ & Spring constant & 0.0093 & $\mathrm{kgm} / \mathrm{s}^{2}$ \\
\hline$g$ & Gravitationally acceleration & 9.82 & $\mathrm{~m} / \mathrm{s}^{2}$ \\
\hline$k_{m}$ & Main motor constant & $74.8 \times 10^{-3}$ & $\mathrm{Nm} / \mathrm{A}$ \\
\hline$k_{t}$ & Tail motor constant & $88.3 \times 10^{-3}$ & $\mathrm{Nm} / \mathrm{A}$ \\
\hline$R_{a m}$ & The ohmic resistance of the main motor & 6.82 & $\Omega$ \\
\hline$R_{a t}$ & The ohmic resistance of the tail motor & 8.05 & $\Omega$ \\
\hline$J_{a m}$ & Total inertia is seen by the main motor & $2.76 \times 10^{-3}$ & $\mathrm{kgm}{ }^{2}$ \\
\hline$J_{a t}$ & Total inertia is seen by the tail motor & $0.5097 \times 10^{-3}$ & $\mathrm{kgm}^{2}$ \\
\hline$B_{a m}$ & Main motor viscous friction constant & $0.346 \times 10^{-3}$ & $\mathrm{kgm}^{2} / \mathrm{s}$ \\
\hline$B_{a t}$ & Tail motor viscous friction constant & $0.250 \times 10^{-3}$ & $\mathrm{kgm}^{2} / \mathrm{s}$ \\
\hline$D_{m}$ & Main motor drag friction constant & $23.03 \times 10^{-6}$ & $\mathrm{kgm}{ }^{2}$ \\
\hline$D_{t}$ & Tail motor drag friction constant & $\mathrm{Ngm} / \mathrm{A}^{*}$ \\
\hline
\end{tabular}

*Note that the drag effect is disregarded when estimating the parameters of the tail rotor, as leaving drag out of the model has no significant influence in the final fit. 